\title{
Synthesis of Bismuth Sodium Titanate Fine Particles with Different Shapes by the Gel-Sol Method
}

\author{
Kiyoshi Kanie ${ }^{1, *}$, Hiroshi Sakai ${ }^{1}$, Junji Tani ${ }^{1}$, Hirofumi Takahashi ${ }^{2}$ and Atsushi Muramatsu ${ }^{1}$ \\ ${ }^{1}$ Institute of Multidisciplinary Research for Advanced Materials, Tohoku University, Sendai 980-8577, Japan \\ ${ }^{2}$ Fuji Ceramics Corporation, Fujinomiya 418-0111, Japan
}

Polycrystalline spherical $\mathrm{Bi}_{0} \mathrm{Na}_{0} \mathrm{TiO}_{3}$ (BNT) particles with narrow size distributions were obtained by the gel-sol method, where the precursory gel was composed of $\mathrm{BiX}_{3}\left(\mathrm{X}=\mathrm{OH}, \mathrm{NO}_{3}\right), \mathrm{NaOH}$, and stable $\mathrm{Ti}^{4+}$ complex which was prepared by mixing $\mathrm{Ti}\left(i-\mathrm{C}_{3} \mathrm{H}_{7} \mathrm{O}\right)_{4}$ with triethanolamine to inhibit the uncontrolled hydrolysis in aqueous solution. The resulting morphology was readily controlled as single-crystalline rod-like and tubular shapes with the simple tuning of the $\mathrm{Bi} / \mathrm{Ti}$ molar ratios in the presence of $\mathrm{NaClO}_{4}$ [doi:10.2320/matertrans.MRA2007043]

(Received February 19, 2007; Accepted May 24, 2007; Published July 19, 2007)

Keywords: perovskite, piezoelectric compound, sodium bismuth titanate, fine particle, gel

\section{Introduction}

Alternative lead-free piezoelectric ceramics, in place of $\mathrm{PbZrTiO}_{3}(\mathrm{PZT})^{1-3)}$ has been investigated, because it evoked problems of human health and environment. Thus, this development has attracted great deals of attention in material sciences. Recently, piezoelectric properties and high Curie temperatures based on bismuth sodium titanate (BNT) derivatives have been considered to be an excellent candidate. ${ }^{4)}$ Intensive efforts have been carried out to improve the characteristic properties of BNT by doping of metal ion species $^{5,6)}$ and changing of the sintering conditions. ${ }^{7)}$ However, such efforts to obtain BNT-based ceramics have been only examined by the solid phase sintering systems, expect for several examples. ${ }^{8,9)}$ In such systems, precise control of the resulting crystallite diameters as well as the grain boundary in nano-scale might be attended with a large difficulty, because most of the accessible powders of the starting materials of BNT have diameters of more than micrometer-order, and mechanically mixed heterogeneous powders were used for the sintering.

In contrast, solution-phase synthesis of such ceramic particles has large potential to obtain high-purity and homogeneous powders controlling with the particle mean sizes, the crystallite diameters, and the morphologies. ${ }^{10,11)}$ Such synthetic systems will be expected to have large advantages to control nano-structures of BNT-based noveltypes of piezoelectric ceramics. We consider to apply the "gel-sol method" to their synthesis system because of its excellent advantages, that is, precise control of size, shape, composition, and structure of monodispersed particles in large quantities, developed by Sugimoto et al., ${ }^{12)}$ which has been applied to the synthesis of monodispersed hematite, ${ }^{13-16)} \mathrm{TiO}_{2},{ }^{17-23)}$ and $\mathrm{BaTiO}_{3}$ particles with perovskitestructure. $^{24)}$

The objective of the present study is to develop an efficient procedure for the preparation of crystalline BNT fine particles in the gel-sol system. Furthermore, we also try to control the shape of BNT by the simple changing of the reaction condition.

*Corresponding author, E-mail: kanie@tagen.tohoku.ac.jp

\section{Experimental}

Unless otherwise noted, all reagents of the highest commercial quality were purchased from Wako pure Chemicals Inc. and were used as received. Water was doubly distilled, deionized, and filtered prior to use. Titanium (IV) tetraisopropoxide (TIPO, 99.9\%) and bismuth hydroxide $\left(\mathrm{Bi}(\mathrm{OH})_{3}\right.$, 99.99\%) purchased form Kojundo Chemical Laboratory Co., Ltd were used for the preparation of bismuth sodium titanate (BNT) nanoparticles. X-ray diffraction (XRD) measurements were carried out on a Rigaku RAD-B system using Ni-filtered $\mathrm{CuK} \alpha$ radiation $(40 \mathrm{kV}, 40 \mathrm{~mA})$ or on a Panalytical X'Pert system. TEM observations were performed by using a JEM-1200EX II and the acceleration voltage was $120 \mathrm{kV}$. High resolution TEM images and energy dispersive X-ray spectra were taken by using a JEOL JEM3010 equipped with a JED-2300T, and the acceleration voltage was $300 \mathrm{kV}$.

The synthetic procedure of BNT was as follows: Initially, a $\mathrm{Ti}^{4+}$ stock solution was prepared by mixing TIPO $(28.4 \mathrm{~g}$, $0.100 \mathrm{~mol}$ ) with triethanolamine (TEOA, $29.8 \mathrm{~g}, 0.200 \mathrm{~mol}$ ) under dry atmosphere to form a stable complex against hydrolysis of $\mathrm{Ti}^{4+}$ on atmospheric conditions. After the resulting pale yellow stock solution consisting of the molar ratio of $[$ TEOA] : [TIPO] $=2: 1$ was kept 1 day at room temperature in a dry air with stirring, water was added to make an aqueous stock solution of $0.50 \mathrm{~mol} \mathrm{dm}^{-3}$ in $\mathrm{Ti}^{4+}$. Then, the $6.0 \mathrm{~mL}$ of the stock solution was mixed with $\mathrm{Bi}\left(\mathrm{NO}_{3}\right)_{3}$ or $\mathrm{Bi}(\mathrm{OH})_{3}(3.0 \mathrm{mmol})$ and $8.0 \mathrm{~mol} \mathrm{dm}{ }^{-3} \mathrm{NaOH}$ solution $(0-6 \mathrm{~mL})$ with stirring. Then, the total volume was adjusted to $12 \mathrm{~mL}$, the resulting white-coloured gel-like materials were further stirred for $10 \mathrm{~min}$ at room temperature. The concentrations of $\mathrm{Ti}^{4+}$ and $\mathrm{Bi}^{3+}$ in the suspension were 0.25 and $0.25 \mathrm{~mol} \mathrm{dm}^{-3}$, respectively, and the $\mathrm{Na}^{+}$ion concentration was adjusted to $2.0 \mathrm{~mol} \mathrm{dm}^{-3}$ by the addition of $\mathrm{NaClO}_{4}$. Then the resulting viscous gel was placed into a Teflon-lined autoclave and aged at $100-250{ }^{\circ} \mathrm{C}$ for $3-24 \mathrm{~h}$ to nucleate and grow the BNT particles. Obtained particles were collected by centrifugation $(18,000 \mathrm{rpm}, 15 \mathrm{~min})$, and the sediments were washed two times with an aqueous $1.0 \mathrm{~mol} \mathrm{dm}^{-3} \mathrm{HNO}_{3}$ solution and three-times with water, respectively, by dispersing followed by centrifuging. The 


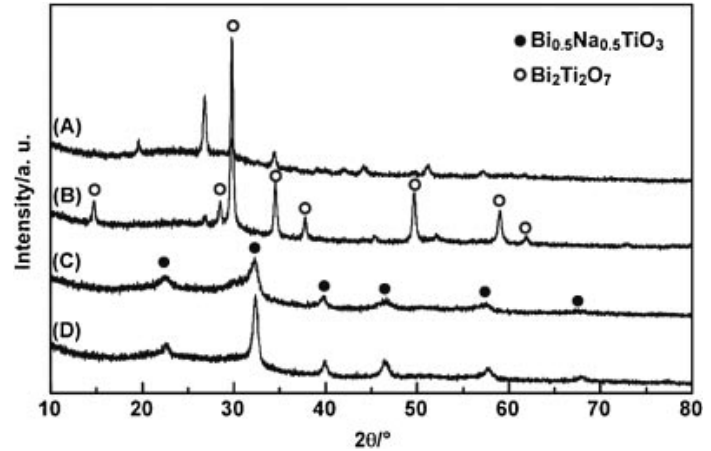

Fig. 1 XRD profiles of solid particles obtained by the changing of the $\mathrm{pH}_{\text {init }}: \mathrm{pH}_{\text {init }}=$ (A) 7.0; (B) 8.5; (C) 13.2; (D) 14.0.

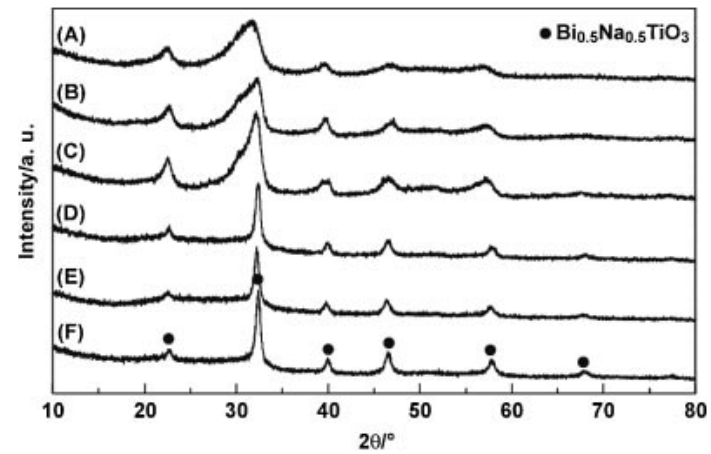

Fig. 3 XRD patterns of solid particles by the changing of the aging temperatures and the periods: (A) $100^{\circ} \mathrm{C}, 3 \mathrm{~h}$; (B) $150{ }^{\circ} \mathrm{C}, 3 \mathrm{~h}$; (C) $200^{\circ} \mathrm{C}$, $3 \mathrm{~h}$; (D) $250^{\circ} \mathrm{C}, 6 \mathrm{~h}$; (E) $250^{\circ} \mathrm{C}, 12 \mathrm{~h}$; (F) $250^{\circ} \mathrm{C}, 24 \mathrm{~h}$.
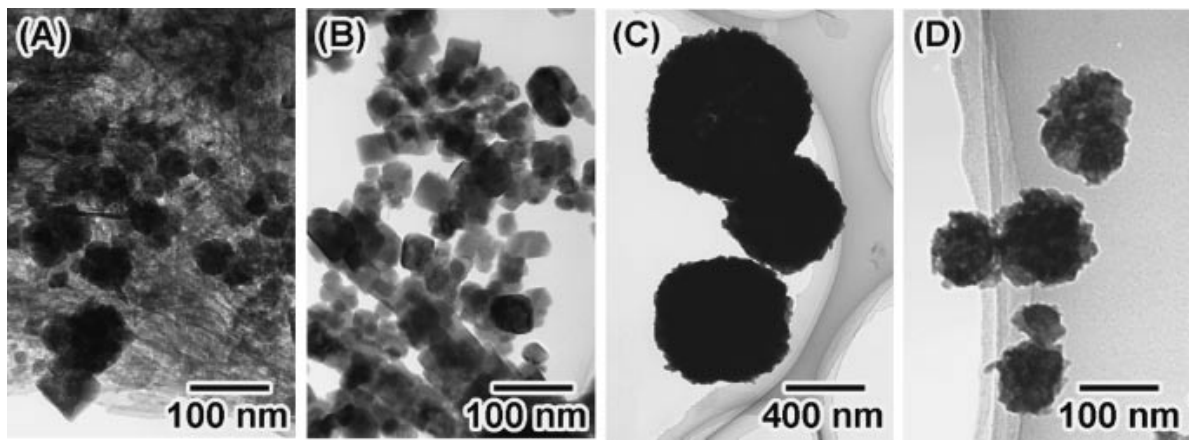

Fig. 2 TEM images of solid particles formed by aging at $250^{\circ} \mathrm{C}$ for $3 \mathrm{~h}: \mathrm{pH}_{\text {init }}=$ (A) 7.0 ; (B) 8.5 ; (C) 13.2 ; (D) 14.0 .

resulting precipitates were dried at $60^{\circ} \mathrm{C}$ to obtain BNT fine particles.

\section{Results and Discussion}

\subsection{The effect of initial pH on the formation of BNT particles}

In general, $\mathrm{pH}$ is a decisive factor on the formation rate of metal oxide particles due to their solubility products of metal ions and hydroxides. ${ }^{10,25)}$ Thus, we investigated to control the crystal structures of the solid particles by changing $\mathrm{pH}$. The initial $\mathrm{pH}\left(\mathrm{pH}_{\text {init }}\right)$ of the mixed solutions of $0.25 \mathrm{~mol} \mathrm{dm}^{-3}$ $\mathrm{Ti}^{4+}$-triethanol amine complex and $0.25 \mathrm{~mol} \mathrm{dm}{ }^{-3} \mathrm{Bi}\left(\mathrm{NO}_{3}\right)_{3}$ $(\mathrm{Bi} / \mathrm{Ti}=1 / 1)$ were adjusted to $7.0,8.0,13.2$, and 14.0 by the addition of $8.0 \mathrm{~mol} \mathrm{dm}^{-3} \mathrm{NaOH}$. The initial concentration of $\mathrm{Na}^{+}$ions was also adjusted to $2.0 \mathrm{~mol} \mathrm{dm}^{-3}$ by varying of additional $\mathrm{NaClO}_{4}$. Figure 1 shows the X-ray diffraction (XRD) profiles of the solid particles aged at $250^{\circ} \mathrm{C}$ for $3 \mathrm{~h}$. Change in $\mathrm{pH}_{\text {init }}$ is strongly influenced to the crystal structure; highly alkaline condition about $\mathrm{pH}_{\text {init }}>13$ is essential for the formation of $\mathrm{BNT}$. Decrease in $\mathrm{pH}_{\text {init }}$ induces the formation of $\mathrm{Bi}_{2} \mathrm{Ti}_{2} \mathrm{O}_{7}$ which was assigned by JCPDS No. 00-032-0118. TEM images of the solid particles obtained with different $\mathrm{pH}_{\text {init }}$ are shown in Fig. 2. Morphologies of the resulting particles are drastically changed by $\mathrm{pH}_{\text {init }}$, cubic and nanometer-level particles are formed at $\mathrm{pH}_{\text {init }}=7.0$ and 8.5, respectively (Fig. 2(a) and (b)). In contrast, spherical particles with the BNT structure, which were also confirmed by energy dispersive X-ray (EDX) analyses, were obtained (Fig. 2(c) and (d)). The particle mean sizes are drastically decreased by increasing $\mathrm{pH}_{\text {init }}$. The surface roughness seems polycrystalline structures. Apparently, crystallite sizes of the BNT particles shown in Fig. 2(c) and (d), estimated by using the Scherrer's equation, ${ }^{26)}$ are about 51 and $25 \mathrm{~nm}$, respectively.

\subsection{The effect of aging temperatures and periods on the crystallinity of BNT particles}

Temperature and time must be a key on size, shape, and crystallinity of BNT. Figure 3 exhibits XRD profiles of BNT particles obtained at $\mathrm{pH}=14.0$ by aging at 100,150 , and $200{ }^{\circ} \mathrm{C}$ for $3 \mathrm{~h}$, and $250^{\circ} \mathrm{C}$ for 6,12 , and $24 \mathrm{~h}$ using $\mathrm{Bi}(\mathrm{OH})_{3}$. The TEM images of the corresponding BNT particles are shown in Fig. 4. When the aging temperatures increased from $100^{\circ} \mathrm{C}$ to $250{ }^{\circ} \mathrm{C}$, the particle mean sizes decreased from ca. $600 \mathrm{~nm}$ to $90 \mathrm{~nm}$ by the increase in nucleus number at the higher aging temperatures. Aging periods also affected the crystallite sizes and the resulting particle shapes. By aging at $250{ }^{\circ} \mathrm{C}$ for $24 \mathrm{~h}$, spherical polycrystalline particles with rough surface are clearly distinguished, and pseudocuboidal particles with smooth surfaces are also formed. The particle mean size of around $100 \mathrm{~nm}$ as a result of TEM observation was larger than the crystallite size of $31 \mathrm{~nm}$, indicating that they are not single-crystalline but polycrystalline.

\subsection{Shape control of BNT particles by the changing of the $\mathrm{Bi} / \mathrm{Ti}$ mixing ratios}

The initial $\mathrm{Bi} / \mathrm{Ti}$ molar ratios strongly influenced the particle morphology. Figures 5 and 6 exhibits TEM images 

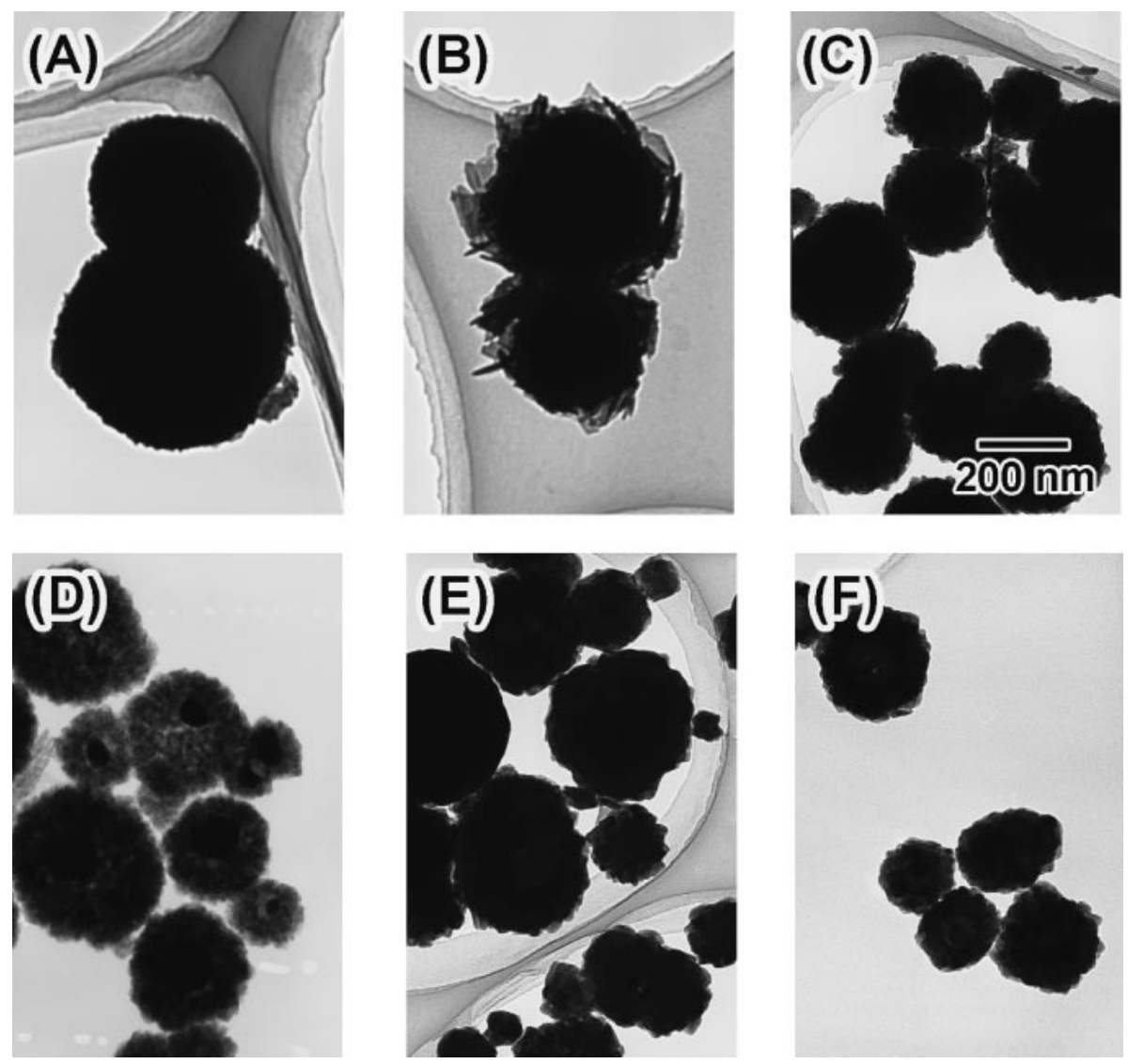

Fig. 4 TEM images of BNT particles by the changing of the aging temperatures and the periods: (A) $100^{\circ} \mathrm{C}, 3 \mathrm{~h}$; $(\mathrm{B}) 150^{\circ} \mathrm{C}, 3 \mathrm{~h}$; (C) $200^{\circ} \mathrm{C}, 3 \mathrm{~h}$; (D) $250^{\circ} \mathrm{C}, 6 \mathrm{~h}$; (E) $250^{\circ} \mathrm{C}, 12 \mathrm{~h}$; (F) $250^{\circ} \mathrm{C}, 24 \mathrm{~h}$.
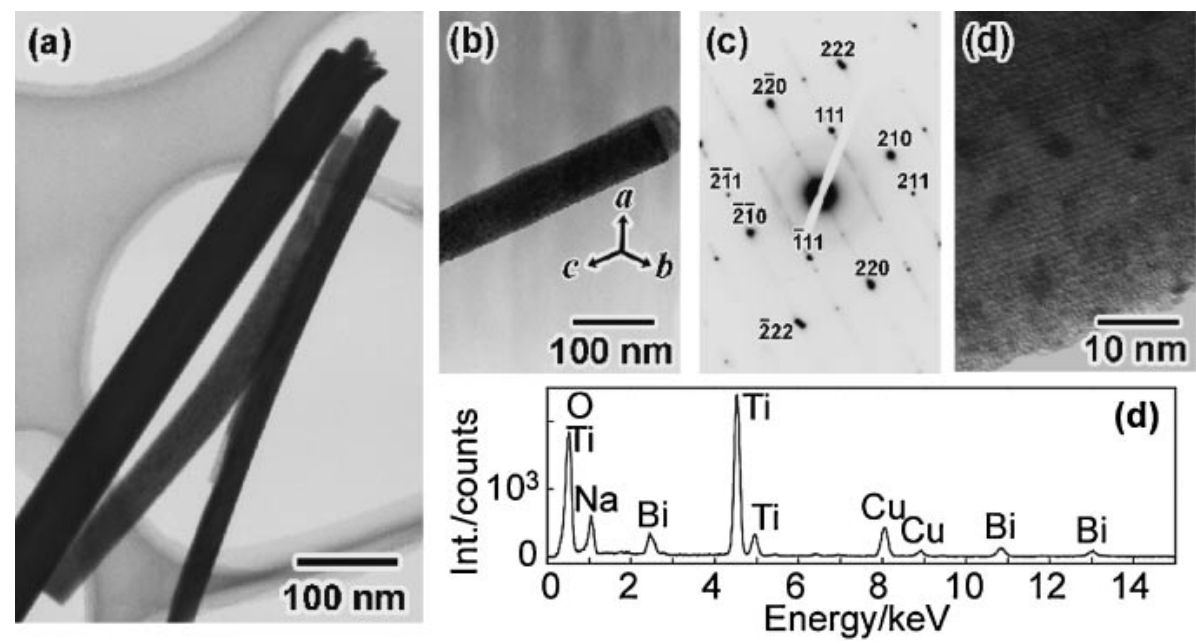

Fig. 5 TEM photographs of (a): BNT nanorods obtained by the changing of Bi/Ti molar ratios $=1 / 2$; (b): a BNT particle and the growth direction determined by ED analyses; (c): an ED pattern obtained from the BNT particle shown in (b); (d): a HRTEM image of the nanorod with lattice fringes; (e): an EDX profile of the BNT nanorods.

of BNT nanorods and nanotubes, respectively, obtained with different initial $\mathrm{Bi} / \mathrm{Ti}$ molar ratios. For $\mathrm{Bi} / \mathrm{Ti}=1 / 2$, singlecrystalline BNT nanorods are formed with a minor diameter ranging from 20 to $200 \mathrm{~nm}$ and a length up to $>10$ microns, as shown in Fig. 5(a). The growth direction of the rod structure (Fig. 5(b)) is determined to be $c$-axis of the perovskitestructure of BNT by the electron diffraction (ED) analysis (Fig. 5(c)). Interestingly, BNT with nanotube-like structures
(Fig. 6(a) and (b)) were obtained in the systems with $\mathrm{Bi} / \mathrm{Ti}=$ 1/4-1/8. ED analysis (Fig. 6(c)) of one particle shown in Fig. 6(b) reveals that the nanotube-like nanoparticles are also obtained by the growth toward the $c$-axis of BNT. EDX analyses (Figs. 5(e) and 6(e)) as well as the XRD measurements of the nanorods and nanotubes also confirmed the formation of BNT structures. Such anisotropic crystal growth toward the $c$-axis to form rod-like or tubular structure has 

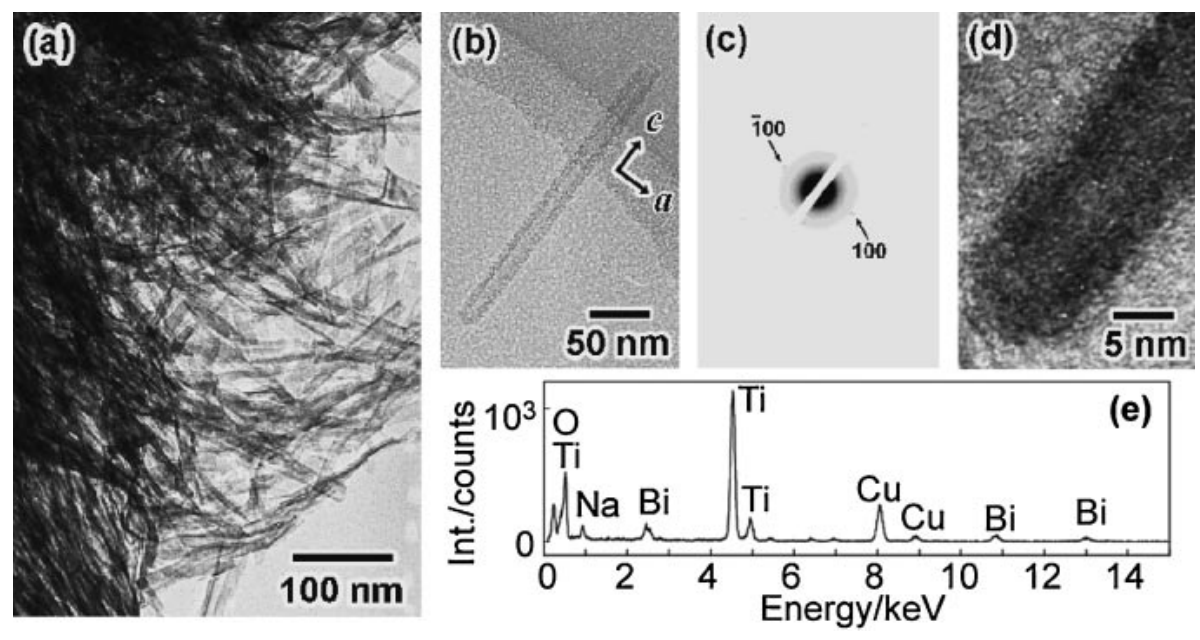

Fig. 6 TEM photographs of (a): BNT nanotubes obtained by the changing of Bi/Ti molar ratios $=1 / 8$; (b): a BNT particle and the growth direction determined by ED analyses; (c): an ED pattern obtained from the BNT particle shown in (b); (d): a HRTEM image of the nanotube with lattice fringes; (e): an EDX profile of the BNT nanotubes.

already been reported by Park $^{27)}$ or Wong ${ }^{28,29)}$ et al., respectively. However, to the best of our knowledge, this is the first report for the morphological control of complex perovskite compounds with various shapes only by controlling the reaction conditions.

The shape control of inorganic nano- and fine-particles progressively increases its importance like other functional materials. ${ }^{10,11,30-35)}$ For example, piezoelectric nanotubes with perovskite-structure are used for the construction of 3D memory devices. ${ }^{36}$ ) The hierarchic 3D structures with high storage density have large potentials for a variety of applications in addition to those in ferroelectric memory devices. Moreover, it has recently been found that novel organic-inorganic hybrid liquid crystals could be fabricated by using monodispersed $\mathrm{TiO}_{2}{ }^{37)}$ or $\alpha-\mathrm{Fe}_{2} \mathrm{O}_{3}{ }^{38)}$ particles with characteristic morphologies. Therefore, it may be readily understood that the precise control of the morphology of functional nanoparticles is one of the most important techniques for the further progress in the modern nanotechnology.

\section{Conclusions}

We have prepared BNT fine particles by using the Gel-Sol method, which is a particle deposition system utilizing highly condensed precursor gels for the preparation of monodispersed particles precisely controlled in size and morphology in large quantities. Main concluding remarks obtained this work are as follows:

(1) Spherical polycrystalline BNT particles were fabricated by the Gel-Sol method starting from highly viscous gels consisting of $0.25,0.25$, and $2.0 \mathrm{~mol} \mathrm{dm}^{-3}$ of $\mathrm{Bi}^{3+}$, $\mathrm{Ti}^{4+}$, and $\mathrm{Na}^{+}$ions, respectively.

(2) The initial $\mathrm{pH}$ of the gels was strongly influenced to the crystal structure of the resulting sediments. Highly alkaline conditions $(\mathrm{pH}>13)$ were essential for the formation of BNT crystal structure.

(3) The aging temperature and the periods played key roles for the resulting particle mean sizes and the crystallite diameters. Higher aging temperature afforded BNT particles with smaller particle mean sizes. Longer aging periods brought the growth of crystallite in the BNT particles.

(4) The morphological control of BNT particles to obtain spherical, rod-like, and tubular shapes have been readily achieved by the simple tuning of the mixing ratios of $\mathrm{Bi} / \mathrm{Ti}$ in the precursor gels.

\section{Acknowledgements}

The authors acknowledge Prof. F. Saito for the substantially useful discussion on BNT synthesis. The authors are grateful to Mr. E. Aoyagi and Mr. Y. Hayasaka, IMR, Tohoku University for high-resolution TEM measurements.

\section{REFERENCES}

1) K. Uchino: Ferroelectric Devices, (Marcel Dekker, New York, 2000) pp. 275-303.

2) K. M. Nair, ed, Ceramic Materials and Multilayer Electric Devices: Proceedings of the High Strain Piezoelectric Materials, Devices and Applications, and Advanced Dielectric Materials and Multilayer Electric Devices Symposia, (Westerville, Ohio, American Ceramic Society, 2004) pp. 253-263.

3) D. C. Lupascu and J. Rödel: Adv. Eng. Mater. 7 (2005) 882-898.

4) V. A. Isupov: Ferroelectrics 315 (2005) 123-147.

5) H. Nagata, M. Yoshida, Y. Makiuchi and T. Takenaka: Jpn. J. Appl. Phys. 42 (2003) 7401-7403.

6) A. Sasaki, T. Chiba, Y. Mamiya and E. Otsuki: Jpn. J. Appl. Phys. 38 (1999) 5564-5567.

7) P. Pookmanee, G. Rujijanagul, S. Ananta, R. B. Heimann and S. Phanichphant: J. Eur. Ceram. Soc. 24 (2004) 517-520.

8) C. Y. Kim, T. Sekino and K. Niihara: J. Am. Ceram. Soc. 86 (2003) 1464-1467.

9) M. M. Lencka, M. Oledzka and R. E. Riman: Chem. Mater. 12 (2000) 1323-1330.

10) T. Sugimoto: Monodispersed Particles, (Elsevier, Amsterdam, 2001) pp. 187-452.

11) Y. Waseda and A. Muramatsu, eds., Morphology Control of Materials and Nanoparticles, (Springer, Berlin, 2003) pp. 25-62.

12) T. Sugimoto: Chem. Eng. Technol. 26 (2003) 313-321.

13) T. Sugimoto, K. Sakata and A. Muramatsu: J. Colloid Interface Sci. 159 (1993) 372-382.

14) T. Sugimoto and K. Sakata: J. Colloid Interface Sci. 152 (1992) 587- 
590.

15) T. Sugimoto, M. M. Khan and A. Muramatsu: Colloid Surf. A 70 (1993) 167-169.

16) T. Sugimoto, S. Waki, H. Itoh and A. Muramatsu: Colloid Surf. A 109 (1996) 155-165.

17) K. Kanie and T. Sugimoto: Chem. Commun. 2004 (2004) 1584-1585.

18) T. Sugimoto, K. Okada and H. Itoh: J. Colloid Interface Sci. 193 (1997) 140-143.

19) T. Sugimoto, K. Okada and H. Itoh: J. Dispersion Sci. Technol. 19 (1998) 143-161.

20) T. Sugimoto, X. Zhou and A. Muramatsu: J. Colloid Interface Sci. 252 (2002) 339-346.

21) T. Sugimoto and X. Zhou: J. Colloid Interface Sci. 252 (2002) $347-$ 353.

22) T. Sugimoto, X. Zhou and A. Muramatsu: J. Colloid Interface Sci. 259 (2003) 43-52.

23) T. Sugimoto, X. Zhou, and A. Muramatsu: J. Colloid Interface Sci. 259 (2003) 53-61.

24) J. Cuya, N. Sato, K. Yamamoto, H. Takahashi and A. Muramatsu: High Temperature Materials and Processes 22 (2003) 197-201.

25) J.-P. Jolivet, C. Chanéac and E. Tronc: Chem. Commun. 2004 (2004) 481-482.

26) B. D. Cullity: Elements of X-ray Diffraction, (Addison-Wesley Pub.,
Reading, Massachusetts, 1978) pp. 81-106.

27) J. J. Urban, W. S. Yun, Q. Gu and H. Park: J. Am. Chem. Soc. 124 (2002) 1186-1187.

28) Y. Mao, S. Banerjee and S. S. Wong: Chem. Commun. 2003 (2003) 408-409.

29) Y. Mao, S. Banerjee and S. S. Wong: J. Am. Chem. Soc. 125 (2003) $15718-15719$.

30) S. Kobayashi, K. Hanabusa, N. Hamasaki, M. Kimura and H. Shirai: Chem. Mater. 12 (2000) 1523-1525.

31) S.-M. Lee, S.-N. Cho and J. Cheon: Adv. Mater. 15 (2003) 441-444.

32) Y.-W. Jun, M. F. Casula, J.-H. Sim, S. Y. Kim, J. Cheon and A. P. Alivisatos: J. Am. Chem. Soc. 125 (2003) 15981-15985.

33) C. J. Murphy and N. R. Jana: Adv. Mater. 14 (2002) 80-82.

34) N. R. Jana, L. Gearheart and C. J. Murphy: Chem. Commun. 2001 (2001) 617-618.

35) J.-T. Hu, L.-S. Li, W.-D. Yang, L. Manna, L.-W. Wang and A. P. Alivisatos: Science 292 (2001) 2060-2063.

36) D. D. Morrison, L. Ramsay and J. F. Scott: J. Phys.: Condens. Matter. 15 (2003) L527-L532.

37) K. Kanie and T. Sugimoto: J. Am. Chem. Soc. 125 (2003) 1051810519.

38) K. Kanie and A. Muramatsu: J. Am. Chem. Soc. 127 (2005) 1157811579. 